\title{
Some examples of first exit times
}

\author{
Jesús Antonio Álvarez López ${ }^{1}$ and Alberto Candel $^{2}$
}

\begin{abstract}
The purpose of this article is to compute the expected first exit times of Brownian motion from a variety of domains in the Euclidean plane and in the hyperbolic plane.
\end{abstract}

\section{Introduction}

The theory of Brownian motion on Riemannian manifolds allows probabilistic interpretations of solutions to second order differential equations on them via the so called Dynkin formula. One example of such equation and solution is the following: if $D$ is a regular domain on a Riemannian manifold $M$ with attending Laplace operator $\triangle$, then the expected value of the "first exit time" from $D$ for Brownian paths in the Wiener space of $M$, if finite, is the minimal solution to the differential equation

$$
\triangle f \equiv-1
$$

on $D$, with $f>0$ on $D$ and $f \equiv 0$ on $\partial D$. The number $\rho(D)=4 \int_{D} f$ is called the torsional rigidity of $D$, a sort of isoperimetric constant whose study originated with Saint-Venant memoir [6].

Ghys [4] gave a spectacular application of first exit times to the topology and dynamics of foliated spaces. The examples and calculations presented here are the base of some exercises in [2, Exercises 2.7.14, C.9.4 and C.9.5], and were motivated by a discussion on Ghys theorem.

\footnotetext{
${ }^{1}$ Departamento de Xeometría e Topoloxía, Facultade de Matemáticas, Universidade de Santiago de Compostela, 15706 Santiago de Compostela, Spain; jesus.alvarez@usc.es ${ }^{2}$ Department of Mathematics, California State University, Northridge, CA 91330, U.S.A.; alberto.candel@csun.edu
} 


\section{Generalities on first exit times and on harmonic functions}

More details about first exit times and probabilistic solutions to differential equations can be found in Dynkin [3]; the theory needed for what follows is detailed in [2, Appendix C].

Consider a Riemannian manifold, $M$, with attending Laplacian $\triangle$. These data permit to construct Brownian motion on $M$, a continuous-time stochastic process taking place in the space of continuous paths $\omega:[0, \infty) \rightarrow M$ that is regulated by a set of probability measures $\left\{P_{x} \mid x \in M\right\}$ (with $P_{x}$ supported on paths $\{\omega(0)=x\}$ ) which are constructed via the heat kernel density of the Laplacian $\triangle$.

Solutions to a variety of differential equations on $M$ admit probabilistic interpretations via Brownian motion. One such example is the following. Let $D \subset M$ be a regular domain (a connected open set with piecewise smooth boundary), and consider the first exit time $T_{D}$ from $D$, the function on paths given by $T_{D}(\omega)=\inf \{t>0 \mid \omega(t) \notin D\}$ (with the standard convention that the infimum of the empty set is $\infty$ ).

The expected first exit with respect to the Brownian measures $\left\{P_{x}\right\}$ defines a function $E_{\bullet}\left[T_{D}\right]: x \mapsto E_{x}\left[T_{D}\right]=\int T_{D}(\omega) \cdot P_{x}(\omega)$ which is 0 for all $x \notin D$, and which is either $E_{x}\left[T_{D}\right]<\infty$ for all $x \in D$, or $E_{x}\left[T_{D}\right] \equiv \infty$ for all $x \in D$.

If $D$ is a relatively compact domain, then $E_{x}\left[T_{D}\right]<\infty$ for all $x \in D$. In this case, Dynkin's formula shows that this function is a solution to the differential equation problem (Saint-Venant problem)

$$
\left\{\begin{array}{cl}
\triangle f \equiv-1 & \text { on } D, \\
f>0 & \text { on } D, \text { and } \\
f \equiv 0 & \text { on } \partial D .
\end{array}\right.
$$

Because of the maximum modulus principle for harmonic functions (to the effect that a function that is harmonic on a relatively compact domain and continuous on its closure must attain its extreme values on the boundary of the domain), the solution to the differential equation (1) is unique: the difference of two solutions is harmonic and equal to 0 on $\partial D$, so it must be 0 on all of $D$. Therefore, if $D$ is relatively compact, the expected first exit time from $D$ is the unique solution to the differential equation (1).

If $D$ is not relatively compact, then $E_{\bullet}\left[T_{D}\right]$ may or may not be a finite function. At any rate, there is an increasing sequence of relatively compact domains $D_{1} \subset D_{2} \subset \ldots \subset D$ that exhaust $D$. The first exit time functions, $T_{n}$, from $D_{n}$ increase pointwise to the first exit time function $T_{D}$ and thus the monotone convergence theorem implies that the sequence of expected first exit times $E_{\bullet}\left[T_{n}\right]$ increases to the expected first exit time $E_{\bullet}\left[T_{D}\right]$. 
Furthermore, if $E_{x}\left[T_{D}\right]<\infty$ for one $x \in D$, then $E_{\bullet}\left[T_{D}\right]<\infty$ everywhere and is a solution to Equation (1). In fact, if the expected first exit time $f=E_{\bullet}\left[T_{D}\right]<\infty$, then $f$ is the minimal solution to that equation on $D$. Indeed, from the above paragraph, you infer that $f=E_{\bullet}\left[T_{D}\right]$ is given by

$$
f=\sup _{B \subset D} f_{B}
$$

where $\{B \subset D\}$ is the set of relatively compact regular domains contained in $D$, and where $f_{B}=E_{\bullet}\left[T_{B}\right]$ is the expected first exit time from $B$. If $g$ is any positive function on $D$ such that $\triangle g=-1$ and $g \equiv 0$ on $\partial D$, then $g \geq f$ because, if that was not the case, then $g<f$ on an open subset of $D$, and so it follows from the definition $f=\sup _{B} f_{B}$ that there exists a relatively compact domain $B \subset D$ where $g<f_{B}$. Then $f_{B}-g$ is harmonic and $>0$ on $B$ but $\leq 0$ on $\partial B$, in contradiction to the maximum modulus principle.

Moving on to a brief review of harmonic functions, besides the already mentioned maximum modulus principle, two other well-known facts will be repeatedly used below. Both concern harmonic functions on domains in $\mathbf{R}^{2}$ endowed with Riemannian metrics conformal to the standard metric, that is, of the form $\varphi(d x \otimes d x+d y \otimes d y)$. The second fact is then that, since the Laplacian for this metric is $\triangle_{\varphi} u=(1 / \varphi)\left(u_{x x}+u_{y y}\right)$, a function $u$ is harmonic on a domain of this type if and only if $u$ is harmonic in the classical sense that $u_{x x}+u_{y y}=0$ all throughout the domain. Because of this, if $\phi$ is a holomorphic function with range in the domain of the harmonic function $u$, then the composite $u \circ \phi$ is harmonic in the domain of $\phi$, as is easily verified via the chain rule, utilizing the harmonicity of $u$ and the Cauchy-Riemann equations for $\phi$.

The third fact about harmonic functions is deeper and concerns their integral representations. To each function $u \geq 0$ that is harmonic on the right half plane $\{x>0\}$ there corresponds a measure $\mu$ on the line $\Im=\{(0, t) \mid$ $-\infty<t<\infty\}$ and a constant $C \geq 0$ so that

$$
u(x, y)=C x+\int_{\Im} \frac{x}{x^{2}+(y-t)^{2}} \cdot \mu(t),
$$

for all $(x, y)$ with $x>0$.

In particular, if $u \geq 0$ is harmonic and extends continuously by 0 to all but finitely many points $t_{1}, t_{2}, \ldots, t_{n}$ on the line $x=0$, then the measure $\mu$ is supported on the set $\left\{t_{k}\right\}$, and $u$ may be expressed as

$$
u(x, y)=C_{\infty} x+\sum_{k=1}^{n} \frac{C_{k} x}{x^{2}+\left(y-t_{k}\right)^{2}},
$$

for some constants $C_{\infty}, C_{1}, C_{2}, \ldots, C_{n} \geq 0$. 


\section{Domains in the Euclidean plane}

In Cartesian coordinates $(x, y) \in \mathbf{R}^{2}$, the Euclidean metric is $d x \otimes d x+d y \otimes d y$, and the Laplacian is given by

$$
\triangle_{e} f=f_{x x}+f_{y y} .
$$

In polar coordinates $(r, \theta)$, the Laplacian is given by

$$
\triangle_{e} f=f_{r r}+\frac{1}{r} f_{r}+\frac{1}{r^{2}} f_{\theta \theta} .
$$

\subsection{Domain bounded by an ellipse}

Let $D$ be the domain enclosed by an ellipse in the Euclidean plane with axes of lengths $a, b>0$ and center $(h, k)$. Up to isometry (a rotation), $D$ consists of all points $(x, y)$ such that $\frac{(x-h)^{2}}{a^{2}}+\frac{(y-k)^{2}}{b^{2}}<1$.

The expected first exit time from $D$ is given by the function

$$
f(x, y)=\frac{a^{2} b^{2}}{2 a^{2}+2 b^{2}}\left(1-\frac{(x-h)^{2}}{a^{2}}-\frac{(y-k)^{2}}{b^{2}}\right) .
$$

Indeed, $f$ is positive on $D$, identically 0 on the ellipse $\partial D$, and satisfies the differential equation $\triangle f=-1$ on $D$. Therefore $E_{(x, y)}\left[T_{D}\right]=f(x, y)$ because, $D$ being relatively compact, Equation (1) has exactly one solution.

\subsection{Domain bounded by a parabola}

Up to isometry, a parabola has an equation of the form $y^{2}=4 p x$ (focus at $(p, 0)$ and focal distance $p$ ), and a convex domain bounded by a parabola is isometric to the domain, $D$, consisting of all $(x, y) \in \mathbf{R}^{2}$ such that $4 p x>y^{2}$.

The expected first exit time from $D$ is

$$
E_{(x, y)}\left[T_{D}\right]=2 p x-\frac{y^{2}}{2},
$$

for all $(x, y) \in D$. 
It is plain that $f(x, y)=2 p x-y^{2} / 2$ is a solution to equation (1) on $D$, but to prove that the expected first exit time $E_{(x, y)}\left[T_{D}\right]=f(x, y)$ on $D$ requires some extra work because $D$ is not relatively compact. Let $D_{n}$, $n>[p]$, denote the domain enclosed by the ellipse with foci at $(p, 0)$ and $(2 n-p, 0)$ and eccentricity $e=1-p / n$. An equation for this ellipse is

$$
\frac{(x-n)^{2}}{n^{2}}+\frac{y^{2}}{2 p(n-p)}=1 .
$$

For $n>[p]$, the domains $D_{n} \subset D_{n+1} \subset \ldots \subset D$ increase to the domain $D$, and so the first exit times functions $T_{n}$ of $D_{n}$ increase pointwise to the first exit time function $T_{D}$. By the dominated convergence theorem, $E_{(x, y)}\left[T_{n}\right]$ converges to $E_{(x, y)}\left[T_{D}\right]$. The expected first exit time from $D_{n}$ was shown to be (4)

$$
\begin{aligned}
E_{(x, y)}\left[T_{n}\right] & =\frac{n^{2} p(n-p)}{2 p(n-p)+n^{2}}\left(1-\frac{(x-n)^{2}}{n^{2}}-\frac{y^{2}}{2 p(n-p)}\right) \\
& =-\frac{p(n-p)}{2 p(n-p)+n^{2}} x^{2}+\frac{2 n p(n-p)}{2 p(n-p)+n^{2}} x-\frac{n^{2}}{4 p(n-p)+2 n^{2}} y^{2} .
\end{aligned}
$$

It follows immediately that $E_{(x, y)}\left[T_{n}\right] \rightarrow 2 p x-y^{2} / 2$, as $n \rightarrow \infty$, which is the expression for the solution to equation (1) shown at (5), uniformly on compact subsets of $D$.

Any solution to Equation (1) is of the form $E_{\bullet}\left[T_{D}\right]+u$, where $u$ is harmonic and $\geq 0$ on $D$ and identically 0 on $\partial D$. The function $\phi: z \mapsto \cosh \frac{\pi}{2} \sqrt{\frac{z}{p}-1}$ is a conformal representation of $D$ onto the right half plane $\Re z>0$. If $u$ is harmonic and $\geq 0$ on $D$ and identically 0 on $\partial D$, then $u \circ \phi^{-1}$ is harmonic and $\geq 0$ on $\Re z>0$ and identically 0 on $\Re z=0$, so, by $(2), u \circ \phi^{-1}(w)=C \Re w$, for some $C \geq 0$, or $u(z)=C \phi(z)$ after the switch $w=\phi(z)$.

That is, any solution to Equation (1) on $D$ is given by (using complex coordinates $z=x+y i$ ):

$$
z \mapsto 2 p \Re(z)-\frac{\Im z^{2}}{2}+C \Re\left(\cosh \frac{\pi}{2} \sqrt{\frac{z}{p}-1}\right),
$$

for some constant $C \geq 0$. 


\subsection{Domain between two concentric circles}

Let $D$ be a domain bounded by two concentric circles of radii $a<b$. Rotations about the common center of the circles are isometries that leave $D$ invariant. Therefore the expected first exit time, $f=E_{\bullet}\left[T_{D}\right]$, from $D$, in polar coordinates $(r, \theta)$ about its center is a function of $r$ only, and so, in those coordinates, the equation $\triangle_{e} f=-1$ becomes, by $(3), f^{\prime \prime}(r)+(1 / r) f^{\prime}(r)=-1$ in $(a, b)$. The general solution is $f(r)=-r^{2} / 4+A \log r+B$, and the boundary conditions $f(a)=f(b)=0$ make $A=\frac{b^{2}-a^{2}}{4(\log b-\log a)}$ and $B=\frac{a^{2} \log b-b^{2} \log a}{4(\log b-\log a)}$.

\subsection{Angular domain}

Let $V \subset \mathbf{R}^{2}$ be an angular domain of angle $\alpha$. In polar coordinates, $V$ is, up to isometry, the set of $(r, \theta)$ with $r>0$ and $\theta \in(-\alpha / 2, \alpha / 2)$.

The expected first exit time from an angular domain $V$ of angle $\alpha$ is infinite if $\alpha \geq \pi / 2$, and is finite if $\alpha<\pi / 2$ and given by

$$
E_{(r, \theta)}\left[T_{V}\right]=\frac{r^{2}}{4}\left(\frac{\cos 2 \theta}{\cos \alpha}-1\right)
$$

Because an angular domain is not a relatively compact domain, there is no guarantee of existence or of uniqueness of solutions to Equation 1. The expected first exit time, $f=E_{\bullet}\left[T_{V}\right]$, from $V$ has two other properties that, if finite, will characterize it uniquely among the solutions to that equation.

(a) The expected first exit time function is homogeneous of order 2. Indeed, for $\lambda>0$, the mapping $(x, y) \mapsto(\lambda x, \lambda y)$ is a dilation of the Euclidean metric that leaves $V$ invariant. The Euclidean heat kernel density at $\lambda p=(\lambda x, \lambda y)$ at time $t$ deposited in $\lambda p^{\prime}=\left(\lambda x^{\prime}, \lambda y^{\prime}\right)$ is the Euclidean heat kernel density at $p=(x, y)$ at time $t / \lambda^{2}$ deposited in $p^{\prime}=\left(x^{\prime}, y^{\prime}\right)$ :

$$
\frac{1}{2 \pi t} e^{-\left|\lambda p-\lambda p^{\prime}\right|^{2} / 4 t} d\left(\lambda x^{\prime}\right) d\left(\lambda y^{\prime}\right)=\frac{1}{2 \pi\left(t / \lambda^{2}\right)} e^{-\left|p-p^{\prime}\right|^{2} / 4\left(t / \lambda^{2}\right)} d x^{\prime} d y^{\prime}
$$

The effect of such dilation is to rescale Brownian motion times by a factor of $\lambda^{2}$, and so the expected first exit time $f$ must satisfy

$$
f(\lambda x, \lambda y)=\lambda^{2} f(x, y)
$$

in polar coordinates

$$
f(\lambda r, \theta)=\lambda^{2} f(r, \theta) .
$$


(b) In polar coordinates, the expected first exit time satisfies $f(r, \theta)=f(r,-\theta)$ because reflection about the axis of $V$ is an isometry that leaves $V$ invariant.

Consequently, by (a), the expected first exit time $f$ is completely determined by the values $f(1, \theta)$, and thus it can be written as $f(r, \theta)=r^{2} h(\theta)$, where $h$ is positive and symmetric on $(-\alpha / 2, \alpha / 2)$. Writing out the differential equation $\triangle_{e} f=-1$ for $f(r, \theta)=r^{2} h(\theta)$ in polar coordinates (3) results in the following differential equation for $h$ on $(-\alpha / 2, \alpha / 2)$ :

$$
h^{\prime \prime}(\theta)+4 h(\theta)=-1 .
$$

The boundary condition $f \equiv 0$ on $\partial C$ results in the boundary condition $h( \pm \alpha / 2)=0$. Note also that $h>0$ and that, by (b), $h$ is symmetric about $0 \in[-\alpha / 2, \alpha / 2]$.

The general solution to Equation (7) is of the form

$$
h(\theta)=A \cos 2 \theta+B \sin 2 \theta-1 / 4 .
$$

The symmetry of $h$ about 0 in $[-\alpha / 2, \alpha / 2]$ implies that $B=0$, and the initial conditions impose that $A=1 /(4 \cos \alpha)$. Therefore,

$$
h(\theta)=\frac{\cos 2 \theta}{4 \cos \alpha}-\frac{1}{4} .
$$

Because this holds for all $\theta$ between $-\alpha / 2$ and $\alpha / 2$, and $h>0$, you must have $\cos \alpha>0$ and with the same sign as $\cos 2 \theta$, and so $\alpha<\pi / 2$. Writing $f(r, \theta)=r^{2} h(\theta)$ confirms the statement at the beginning of this section.

In rectangular coordinates, an angular domain of angle $\alpha<\pi / 2$ is isometric to the domain, $V$, consisting of all $(x, y)$ such that $x>m|y|$, where $0<m=\tan \alpha / 2<1$.

The expected first exit time from $V=\{(x, y)|x>m| y \mid\}$, with $0<$ $m<1$, is

$$
E_{(x, y)}\left[T_{V}\right]=\frac{1}{2-2 m^{2}}\left(m^{2} x^{2}-y^{2}\right)
$$

Any solution, $g$, to Equation (1) on $V$ is given by

$$
g(x, y)=\frac{1}{2-2 m^{2}}\left(m^{2} x^{2}-y^{2}\right)+C\left(x^{2}+y^{2}\right)^{\pi / \alpha} \cos \frac{\pi \arctan (y / x)}{\alpha},
$$

for some constant $C \geq 0$.

The second summand in the expression for $g$ is justified in a manner similar to that of the case of the parabola at the end Section 3.2. In this case, you 
consider the conformal representation, $\phi$, of the angular domain $V$ above onto the right half plane given by $\phi(z)=z^{\pi / \alpha}$, and then you use (2) to show that any non-negative harmonic function on $V$ that is identically 0 on $\partial V$ is of the form $z \mapsto C \Re\left(z^{\pi / \alpha}\right)$, which is as stated above.

\subsection{Domain bounded by a hyperbola}

\subsubsection{Convex domain}

Let $D$ be a convex domain in $\mathbf{R}^{2}$ bounded by a hyperbola. Up to isometry, this hyperbola is given by an equation of the form $(x / a)^{2}-(y / b)^{2}=1$, with $a, b>0$, and $D$ consists of all $(x, y)$ such that $(x / a)^{2}-(y / b)^{2}>1$ and $x>0$.

If $b \geq a$, then the expected first exit time from $D$ is infinite.

Indeed, $D$ contains the angular domain $V$ bounded by the lines $y=$ $\pm m(x-a)$ with $m=b / a$ and $x>a$, and so the expected first exit times $E_{\bullet}\left[T_{D}\right] \geq E_{\bullet}\left[T_{V}\right]$. If $b \geq a$, then $V$ has angle $2 \arctan m \geq \pi / 2$, and so, as established in Section 3.4, the expected first exit time from $V$ is infinite.

If $b<a$, then the expected first exit time from $D$ is given by the function

$$
g(x, y)=\frac{1}{2-2 m^{2}}\left(m^{2} x^{2}-y^{2}-b^{2}\right),
$$

for all $(x, y) \in D$.

It is plain that $g$ is a solution to Equation (1). If $g$ is not the expected first exit time from $D$, then it is not the minimal solution to (1), and so the expected first exit time from $D$ is of the form $E_{\bullet}\left[T_{D}\right]=g-u$, where $u$ is a positive, harmonic function on $D$ satisfying $u \equiv 0$ on $\partial D$.

If $m=b / a<1$, the expected first exit time $E_{\bullet}\left[T_{V}\right]$ is finite and given by (8) (after a horizontal shift), and is a minorant for $E_{\bullet}\left[T_{D}\right]=g-u$. Thus

$$
\left.\frac{1}{2-2 m^{2}}\left(m^{2}(x-a)^{2}-y^{2}\right)\right) \leq \frac{1}{2-2 m^{2}}\left(m^{2} x^{2}-y^{2}-b^{2}\right)-u(x, y),
$$

or

$$
u(x, y)<\frac{b^{2}}{1-m^{2}}\left(\frac{x}{a}-1\right)
$$

for all $(x, y)$ in $V$. 
You will now reach a contradiction as follows. The function $\phi$ given by (appropriate branches taken)

$$
\begin{aligned}
\phi(z) & =c \cosh \left(\frac{2 \mu}{\pi} \operatorname{arcosh} z\right) \\
& =\frac{c}{2}\left(\left(z+\sqrt{z^{2}-1}\right)^{2 \mu / \pi}+\left(z+\sqrt{z^{2}-1}\right)^{-2 \mu / \pi}\right),
\end{aligned}
$$

where $\mu=\arctan m$ and $c=\sqrt{a^{2}+b^{2}}$ the linear eccentricity, is a conformal representation of the right half plane $\{x=\Re z>0\}$ onto $D$ which takes the boundary $\{x=\Re z=0\}$ onto $\partial D$ and the ray $\{x \geq 0\}$ onto the ray $\{x \geq a\}$. (As an aid in visualizing this mapping, you recall that cosh takes the horizontal line through $\beta i(0<\beta<\pi / 2)$ onto the right branch of the hyperbola of equation $x^{2} / \cos ^{2} \beta-y^{2} / \sin ^{2} \beta=1$, cf. [1, 3.4.2] or [5] for more background.)

Then, on the one hand, you deduce from inequality (9) that

$$
u(\phi(x))<\frac{b^{2}}{1-m^{2}}\left(\frac{\phi(x)}{a}-1\right),
$$

because $\phi(z)$ is real for $z$ real, and from this inequality that $\lim _{x \rightarrow \infty} \frac{u(\phi(x))}{x}=0$, because, for real $x>1, \phi(x)=\frac{c}{2}\left(\left(x+\sqrt{x^{2}-1}\right)^{2 \mu / \pi}+\left(x+\sqrt{x^{2}-1}\right)^{-2 \mu / \pi}\right)$, hence $\phi(x)=O\left(x^{2 \mu / \pi}\right)$, and so $\lim _{x \rightarrow \infty} \frac{\phi(x)}{x}=0$ because $\mu=\arctan m<\pi / 2$.

On the other hand, the composite function $u \circ \phi$ is positive and harmonic on $\Re z>0$ and is identically 0 on the boundary $\Re z=0$. Therefore, by (2), $u \circ \phi(x, y)=C x$, for some constant $C>0$, and so $\lim _{x \rightarrow \infty} \frac{u(\phi(x))}{x}=C>0$.

\subsubsection{Concave domain}

A (concave) domain bounded by the two branches of a hyperbola is isometric to the domain $D=\left\{(x, y) \in \mathbf{R}^{2} \mid x^{2} / a^{2}-y^{2} / b^{2}>-1\right\}$, for some $a, b>0$. Let $m=b / a$ and $\mu=\arctan m$. If $m \geq 1$, then the expected first exit time from $D$ is infinite because $D$ contains an angular domain of angle $2 \mu \geq \pi / 2$.

If $b<a$, the expected first exit time from $D$ is given by the function

$$
g(x, y)=\frac{1}{2-2 m^{2}}\left(m^{2} x^{2}-y^{2}+b^{2}\right),
$$

for all $(x, y) \in D$. 
Certainly, $g$ is a solution to Equation (1) on $D$, so $g=E_{(x, y)}\left[T_{D}\right]+v$, where $v$ is $\geq 0$ and harmonic on $D$ and extends continuously to 0 on $\partial D$.

The domain $D$ contains the angular domains $V_{-}=\left\{x<0 \& b^{2} x^{2}>\right.$ $\left.a^{2} y^{2}\right\}$ and $V_{+}=\left\{x>0 \& b^{2} x^{2}>a^{2} y^{2}\right\}$. These domains have angle $2 \mu<\pi / 2$, so their expected first exit time is finite and given by $f_{ \pm}(x, y)=$ $\frac{a^{2} b^{2}}{2 a^{2}-2 b^{2}}\left(b^{2} x^{2}-a^{2} y^{2}\right)$ (same expression, different domain). By comparison,

$$
\frac{a^{2} b^{2}}{2 a^{2}-2 b^{2}}\left(b^{2} x^{2}-a^{2} y^{2}\right) \leq \frac{a^{2} b^{2}}{2 a^{2}-2 b^{2}}\left(b^{2} x^{2}-a^{2} y^{2}+1\right)-v(x, y),
$$

or,

$$
v(x, y) \leq \frac{a^{2} b^{2}}{2 a^{2}-2 b^{2}},
$$

for all $(x, y) \in V_{-} \cup V_{+}$. In particular, $v$ is bounded on the real axis $\{y=0\}$.

The mapping $\psi$ given by

$$
\begin{aligned}
\psi(z) & =\frac{c}{2}\left(z^{2 \mu / \pi}-\frac{1}{z^{2 \mu / \pi}}\right) \\
& =c \sinh \left(\log z^{2 \mu / \pi}\right),
\end{aligned}
$$

where $c=\sqrt{a^{2}+b^{2}}$, is a conformal representation of the right half plane $\Re z>0$ onto $D$ that takes the positive imaginary axis $\Im z>0$ to the upper branch of the hyperbola bounding $D$, takes the negative imaginary axis to the lower branch of that hyperbola, and takes the positive real axis onto the real axis (cf. [1, 3.4.2] or [5] for help on constructing this mapping). Therefore, the composite function $v \circ \psi$ is a positive, harmonic function on $\Re z>0$ and that extends continuously by 0 to $\Im z \neq 0$ on the right half plane. Therefore, by (2), $v \circ \psi$ is of the form

$$
v \circ \psi(z)=A \Re z+B \Re(1 / z)
$$

for some constants $A, B \geq 0$. Because of (11), the composite $v \circ \psi$ must be bounded on the positive real axis $\Im z=0$, and therefore both $A=0$ and $B=0$, that is, $v \equiv 0$, which shows that the expected first exit time $E_{\bullet}\left[T_{D}\right]=g$ as was stated at $(10)$.

\section{Domains in the Hyperbolic Plane}

The unit disk model for the hyperbolic plane is realized by the unit disk $\left\{x^{2}+\right.$ $\left.y^{2}<1\right\}$ in $\mathbf{R}^{2}$, endowed with the metric $4\left(1-x^{2}-y^{2}\right)^{-2}(d x \otimes d x+d y \otimes d y)$. 
In geodesic polar coordinates $(r, \theta)$ about a point, the hyperbolic metric is $d r \otimes d r+\sinh ^{2} r d \theta \otimes d \theta$, and the corresponding Laplacian is

$$
\triangle_{h} f=f_{r r}+2 \operatorname{cotanhr} f_{r}+f_{\theta \theta} .
$$

The right half plane model for the hyperbolic plane is realized on the domain $\{x>0\}$ with the metric $x^{-2}(d x \otimes d x+d y \otimes d y)$, with Laplacian

$$
\triangle_{h} f=x^{2}\left(f_{x x}+f_{y y}\right) .
$$

In Euclidean polar coordinates $(r, \theta)$, with $r>0$ and $-\pi / 2<\theta<\pi / 2$, the Laplacian is

$$
\triangle_{h} f=\left(\cos ^{2} \theta\right)\left(r^{2} f_{r r}+r f_{r}+f_{\theta \theta}\right)
$$

\subsection{Hyperbolic disks}

Let $D$ be a disk of radius $R$ in the hyperbolic plane. In geodesic coordinates $(r, \theta)$ based at the center of $D$, the Laplacian is $\triangle_{h} f=f_{r r}+2 \operatorname{cotanhr} f_{r}+f_{\theta \theta}$. The expected first exit time from $D$ is invariant by (hyperbolic) rotations about the center of $D$, therefore $f=f(r)$ is a function of the distance $r$ only (the hyperbolic distance to the center of the disk), and so the equation $\triangle_{h} f=-1$ on $D$ becomes, by (12),

$$
f^{\prime \prime}(r)+2 \operatorname{cotanh}(r) f^{\prime}(r)=-1
$$

on $(0, R)$, with the boundary conditions that $\lim _{r \rightarrow 0} f(r)$ exists and that $f(R)=$ 0 . The general solution is $f(r)=-(r / 2) \operatorname{cotanh} r+A \operatorname{cotanh} r+B$, and the boundary conditions imply that $A=0$ and that $B=(R / 2) \operatorname{cotanh} R$.

The expected first exit time from a hyperbolic disk of radius $R$ is

$$
f(r, \theta)=-\frac{r}{2} \operatorname{cotanh} r+\frac{R}{2} \operatorname{cotanh} R .
$$

\subsection{Horodisks}

A horodisk is a domain in the hyperbolic plane that may be visualized as a hyperbolic disk with center at a point on the ideal boundary of the hyperbolic 
plane. Any horodisk is isometric to the domain, $D$, in the right half plane model consisting of the points $(x, y)$ with $x>R$, for some constant $R>0$.

The expected first exit time from the horodisk $D=\{x>R\}$ is given by

$$
E_{(x, y)}\left[T_{D}\right]=\log \frac{x}{R}
$$

The expected first exit time, $f=E_{\bullet}\left[T_{D}\right]$, from $D$ must be invariant under vertical translations because these are hyperbolic isometries that leave $D$ invariant; that is, $f(x, y+t)=f(x, y)$ for all $t$, or $f(x, y)=f(x)$ for all $y$. Given this, the equation $\triangle_{h} f=-1$, with $f \equiv 0$ on $x=R$, reduces to

$$
f^{\prime \prime}(x)=-x^{-2}
$$

on $(R, \infty)$, with $f(R)=0$, that is $f(x, y)=\log \frac{x}{R}$, as advertised at (16).

\subsection{Neighborhoods of geodesics}

A neighborhood, $D$, of a geodesic in the hyperbolic plane is determined, up to isometry, by its radius: $D=D(R)$. In the right half plane model and in Euclidean polar coordinates $(r, \theta)$ as in (14), the geodesic is $\theta=0$, and $D(R)$ is the set of all $(r, \theta)$ with $r>0$ and $-\alpha<\theta<\alpha$, where $\log \frac{\cos \alpha}{1-\sin \alpha}=R$.

Because the mapping $(r, \theta) \mapsto(\lambda r, \theta)$ is an isometry of the hyperbolic plane that preserves $D$ (it is a hyperbolic translation along the geodesic $\theta=0$ ), the expected first exit time, $f=E_{\bullet}\left[T_{D}\right]$, must satisfy $f(r, \theta)=f(\lambda r, \theta)$ for all $\lambda>0$. That is, $f(r, \theta)=f(\theta)$ is a function of $\theta$ only. So writing out the equation $\triangle_{h} f=-1$ in coordinates $(r, \theta)$, results in, cf. (14),

$$
f^{\prime \prime}(\theta)=\frac{-1}{\cos ^{2} \theta} \text {. }
$$

The general solution is $f(\theta)=\log \cos \theta+A \theta+B$. The expected first exit time is invariant under reflection about $\theta=0$, so the constant $A=0$, and the boundary condition $f(\alpha)=0$ forces $B=-\log \cos \alpha$.

The expected first exit time from $D=D(\alpha)$ is given by

$$
f(r, \theta)=\log \frac{\cos \theta}{\cos \alpha} \text {. }
$$


Any solution to Equation (1) on $D$ is of the form

$$
f(r, \theta)+\left(A r^{\pi / 2 \theta}+\frac{B}{r^{\pi / 2 \theta}}\right) \cos \frac{\pi \theta}{2 \alpha},
$$

for some constants $A, B \geq 0$.

Indeed, the composite of a non-negative harmonic function, $u$, on $D$ that is 0 on the boundary $\partial D$ and the conformal representation of the right half plane $\{x>0\}$ onto $D$ given by $\phi:(r, \theta) \mapsto\left(r^{2 \alpha / \pi}, 2 \alpha / \pi \theta\right)$ is a non-negative harmonic function, $u \circ \phi$, on the right half plane that is identically 0 on the boundary $\theta= \pm \pi / 2$, except perhaps at the origin. By (2), such function is of the form $(r, \theta) \mapsto(A r+B / r) \cos \theta$, for some constants $A, B \geq 0$.

\subsection{Neighborhood of ideal point}

You may adjust the calculation in the previous section to cover the case of a one-sided neighborhood of a geodesic. In Euclidean polar coordinates as above, the one-sided neighborhood of radius $R$ is isometric to the domain $D=D(R)$ of all $(r, \theta)$ with $r>0$ and $0<\theta<\alpha(R)$, with $\alpha(R)$ as in the first paragraph of Section 4.3. The expected first exit time from $D(R)$ is

$$
E_{(r, \theta)}\left[T_{D(R)}\right]=\log \cos \theta-\frac{\theta}{\alpha} \log \cos \alpha .
$$

A neighborhood of an ideal point is isometric to the domain $D(R)$ when $R=\infty$, or $\alpha=\pi / 2$. Hence, the expected first exit time from such domain is infinite because there is no positive solution to the differential equation $f^{\prime \prime}(\theta)=-1 / \cos ^{2} \theta$ on $(0, \pi / 2)$ with boundary conditions $f(0)=f(\pi / 2)=0$.

\section{References}

1. Lars Ahlfors, Comples Analysis, (3rd edition), International Series inPure and Applied Mathematics, McGraw-Hill, Inc, New York, 1979.

2. Alberto Candel and Lawrence Conlon, Foliations II, Graduate Studies in Mathematics, Vol. 60, Amer. Math. Soc., Providence, RI, 2003.

3. Eugene Dynkin, Markov Processes. Grundlehren der mathematischen Wissenschaften Vols. 121, 122, Springer, Berlin, 1965.

4. Étienne Ghys, Topologie des feuilles génériques. Ann. Math. 141 (1995), pp. 387-422

5. Hermann Kober, Dictionary of Conformal Representations, Dover Publications, New York, 1952.

6. Adhémar J.-C. Barre de Saint-Venant, Mémoire sur la torsion des prismes, Mémoires présentés par divers savants à l'Académie des Sciences, 14 (1856), pp. 233-560. 